Leading Article

\title{
Current views on the aetiology and management of the irritable bowel syndrome
}

\author{
Michael J. Hall* and Ralph E. Barry
}

University Department of Medicine, Bristol Royal Infirmary, Bristol BS2 $8 \mathrm{HW}, \mathrm{UK}$

\section{Introduction}

Functional disorders of the gastrointestinal tract may account for nearly $50 \%$ of patients seen by a gastroenterologist. ${ }^{1}$ Many of these will have the classical 'painful variant' of the irritable bowel syndrome (IBS) which Thompson has defined as the presence of abdominal pain and altered bowel habit (constipation or diarrhoea or both in alternation) in the absence of positive findings on physical investigation. ${ }^{2}$ Six cardinal symptoms have been identified: abdominal pain relieved by bowel action, more frequent or looser stools with the onset of pain, visible abdominal distension, the rectal passage of mucus and a sensation of incomplete evacuation of the rectum after defaecation. ${ }^{3}$ The finding of clusters of these symptoms in two independent groups of women drawn from the community supports the existence of IBS as a distinct syndrome. ${ }^{4}$ Questionnaires administered to healthy subjects have indicated that approximately $15 \%$ have symptoms compatible with IBS and that many of these had never consulted a doctor for their bowel complaints. ${ }^{5,6}$ This suggests that psychological and sociocultural factors play an important part in the help-seeking behaviour of some patients with IBS.

\section{Psychological factors}

Indeed, a high prevalence of psychological symptoms and psychoneurotic personality traits has been identified in IBS patients compared to healthy controls. $^{7,8}$ But when comparisons are made between IBS sufferers who seek medical care and those who do not, opinions are divided. Welch and colleagues ${ }^{9}$ found no psychological differences between the two groups but others have shown that the 'patients' exceeded the 'non-patients' in their degree of depression, hypochondriasis and hysteria

Correspondence: R.E. Barry, B.Sc., M.D., F.R.C.P.

*Present address: County Hospital, Hereford HR1 2ER, UK.

Received: 22 March 1991 or in their frequency of learned illness behaviour. ${ }^{10,11}$ Patients with IBS or lactose malabsorption had more psychological symptoms than subjects with the same diagnosis who had not sought medical attention, suggesting that it is the psychological distress which influences which patient consults a doctor. ${ }^{12}$ Furthermore, women 'non-patients' with IBS symptoms had no more psychological symptoms than asymptomatic controls. $^{12}$

Despite this body of evidence demonstrating psychological determinants of health-seeking behaviour, there is also evidence that IBS patients have more severe symptoms than 'non-patients'. ${ }^{10,13}$ Out of 54 persons with IBS, every single symptom or measurement of bowel dysfunction was worse in the 27 patients compared to the 27 non-complainers. ${ }^{13}$ It has been known for many years that IBS patients have a reduced tolerance for balloon distension of the rectosigmoid ${ }^{14}$ but this does not appear to be due to any psychological tendency to exaggerate the painfulness of any aversive stimulus. IBS patients were just as tolerant of holding a hand in iced water than control subjects ${ }^{15}$ and more tolerant of electrocutaneous stimulation. ${ }^{16}$ Furthermore, there was no correlation of rectal balloon tolerance with any psychological trait. ${ }^{15}$ Infusion of an inert gas into the gut produced greater discomfort in IBS patients than in normal subjects ${ }^{17}$ and recent studies have shown that rectal sensitivity was a particular feature of patients with predominant diarrhoea. ${ }^{18}$ Thus a peripheral mechanism, such as altered receptor sensitivity, may be the cause of distension pain in some patients with IBS. Stressful life events have been correlated with the development of IBS but it seems more likely that the life events were the precipitant of medical consultation. ${ }^{19}$

\section{Motility}

It is generally assumed that IBS is related to disordered gut motility, particularly of the colon. 
However, there is no good evidence for a consistent disorder of colonic physiology. Initial studies suggested that a 3 cycle/minute pattern of myoelectrical activity might distinguish IBS patients ${ }^{20}$ but subsequent studies did not confirm this. ${ }^{21} \mathrm{~A}$ recent study of distal colonic intraluminal pressure attempted to produce as little disturbance as possible in placing the pressure transducers and found increased pressure wave amplitude and activity but not duration in IBS patients compared to controls both pre- and postprandially. ${ }^{22}$

The patterns of normal small intestinal motility are much more established and studies of patient groups are therefore much more reliable. ${ }^{23}$ Two specific patterns of small bowel motor activity have been shown to be more common in IBS patients, ileal propulsive waves and irregular contractile activity in the upper jejunum. ${ }^{24,25}$ Both patterns of activity have been shown to occur synchronously with abdominal pain in some patients.

\section{Stress and motility}

Although epidemiological studies indicate that there is an association between stressful experiences and disturbed bowel function, ${ }^{26}$ it has been much more difficult to demonstrate a consistent stress-induced pattern of dysmotility in IBS patients. ${ }^{27}$ Reduction in frequency of small intestinal migratory motor complexes induced by experimental psychological stress has been observed in addition to the occurrence of abnormal irregular contractions ${ }^{24}$ but they have also been observed in the absence of stress. ${ }^{25}$

Increased colonic pressure activity in IBS patients in response to acute stress was demonstrated in the elegant studies of Almy and coworkers ${ }^{28}$ but similar findings have been seen in normal subjects. ${ }^{29}$

\section{IBS subgroups}

The variability of presentation implies that IBS may include several distinct conditions and certain subgroups, especially where significant diarrhoea occurs, have already been identified. The use of radiolabelled bile acids has demonstrated idiopathic malabsorption of bile acids in some patients. ${ }^{30}$ Others, predominantly with painless diarrhoea, improve when certain foods, particularly wheat, are excluded from their diet. ${ }^{31}$ There is no evidence that pain in IBS patients results from bacterial fermentation of unabsorbed carbohydrate ${ }^{32}$ but lactase deficiency may result in symptoms of IBS in a few susceptible patients. ${ }^{33}$

\section{Hypothesis}

Despite the continued failure to identify a single $\stackrel{\mathbb{D}}{\stackrel{2}{2}}$ pathognomonic abnormality in IBS, a consensus $C$ viewpoint on likely aetiological mechanisms $\overrightarrow{\vec{F}}$ appears to be emerging. ${ }^{33-36}$ The enteric nervous $\stackrel{\text { ? }}{+}$ system has many of the characteristics of brain tissues - origin in the neural crest, richness in $\frac{\bar{\sigma}}{\bar{\omega}}$. internuncial neurones, similar synaptic chemistry $-\frac{\bar{\rho}}{\bar{\sigma}}$ and it is connected to the central nervous system $\stackrel{\mathbb{Q}}{\circ}$ (CNS) by far more afferent fibres than efferent $\%$ fibres. The enteric nervous system or 'gut brain" ${ }^{35} \overrightarrow{0}$ integrates sensory information from mucosal receptors and organizes an appropriate motor $\vec{\omega}$ response from a choice of predetermined program- $\frac{\text { S }}{8}$ mes. These enteric reflexes may be sensitized by increased activity in vagal efferent nerves. Such increased activity emanating from the CNS may be $\stackrel{\text { ? }}{ }$ related to psychological factors such as stress, $\vartheta$ anxiety, or life events and lead to hypersensitivity to both neurohumeral or mechanical stimuli with resultant dysmotility. ${ }^{36}$

\section{Fibre}

Manning and co-workers reported significant improvement in symptoms of IBS by the addition of $7 \mathrm{~g}$ fibre daily given in the form of wheat bran More recently, in a double-blind crossover triak bran biscuits were found to be no better that placebo although both groups improved on the first 3 months of treatment. ${ }^{38}$ Symptomatic response in those in whom stool weights rose on bran was no different from those in whom stool weights fell, suggesting that the effect of the fibre supplements is due to a placebo response. ${ }^{38} \mathrm{~A}$ strong placebo response has also been found with bulking agents such as ispaghula with no significant improvement over placebo being obtained in abdominal pain or bloating. ${ }^{39}$ Both bran and ispaghula significantly improve constipation..$^{39,40}$ Rectosigmoid pressures which were found to correlate with symptom severity were not significantly altered by fibres or placebo although there was a trend towards reduction after fibre. ${ }^{41}$

\section{Drugs}

Although bulking agents and anticholinergic antispasmodics are perhaps the most frequently used preparations, the wide variety of other drugs that have been tried in IBS reflects the inefficacy of treatment in many patients. In 1988 all randomized placebo-controlled double-blind trials of the symptomatic treatment of IBS were reviewed. ${ }^{42}$ In addition to antispasmodics and bulking agents, trials of antidepressants, dopamine agonists, car- 
minatives, opioid antidiarrhoeals and tranquillizers were examined and it was concluded that not a single study offered convincing evidence of efficacy in treating IBS. One of the criticisms of the reviewed studies was their short duration. More recently a longer term study over 6 months examined the effect of a new anticholinergic drug, cimetropium bromide, and found that it was significantly better than placebo in improving symptoms..$^{43} \mathrm{An}$ initial report of success in controlling functional bowel symptoms in $\mathbf{4}$ female patients by leuprolide acetate, a gonadotrophin-releasing hormone analogue, is worthy of follow up..$^{44}$ Creed and Guthrie have recently reviewed the role of tranquillizers and antidepressant drugs in IBS and concluded that the current literature does not answer the question whether such drugs can improve bowel symptoms secondary to an alteration of mood state. ${ }^{45}$ It is possible that psychotropic drugs may improve symptoms of IBS because of their analgesic properties and direct effect on bowel motility. ${ }^{45}$

\section{Psychological treatments}

\section{Psychotherapy}

Psychotherapy produced significant improvement in IBS symptoms in $78 \%$ patients as assessed by a self administered questionnaire 8-33 months later, in an uncontrolled trial of 60 consecutive patients. ${ }^{46}$ A later study showed that psychotherapy, along with antispasmodics and bulking agents, resulted in a greater improvement in bowel symptoms and adbominal pain than in a control group which did not receive psychotherapy. ${ }^{47}$ Interestingly, there was no difference in the two groups in the effect on their, albeit mild, psychological symptoms. Although psychotherapy depends for its success on development of a trusting relationship with the therapist it is not just a question of spending time with a patient as is shown by the lack of benefit in Whorwell's control group in a study of hypnosis versus 'psychotherapy'. ${ }^{48}$ In a recent randomized controlled trial of 102 patients with IBS unresponsive to medical treatment, psychotherapy resulted in significant improvement in psychological and gastrointestinal ratings compared to the control group which received 'supportive listening' only. ${ }^{49}$

\section{Behaviour therapy}

Behaviour therapy in which patients are taught exercises or strategies to aid symptom control along with relaxation has been shown to be just as good as medical treatment as a first line management approach. ${ }^{50}$ Similar results were reported by Blanchard and colleagues who identified anxiety, if severe, as a predictor of poor response although milder degrees of anxiety were helped. ${ }^{51}$

\section{Hypnotherapy}

The high placebo response in IBS exacerbates the difficulty of evaluating new treatments in this condition. It is of great interest therefore that Whorwell and co-workers reported on the value of hypnosis in patients who had been refractory to other forms of treatment. ${ }^{48}$ Thirty patients were randomly allocated to receive either seven 30minute sessions of 'gut directed' hypnotherapy or an equivalent time of supportive psychotherapy (although this was carried out by an untrained psychotherapist). In addition, the patients were given a tape for daily autohypnosis. By 3 months the overall changes in abdominal pain, bowel habit, abdominal distension and wellbeing were significantly better in the hypnosis group than in the control subjects. ${ }^{48}$ Further experience and followup indicate that response is well maintained or can be quickly regained with further hypnosis. ${ }^{52}$ The recruitment of more patients has shown that patients who are under 50 years of age or who have 'classical' IBS respond better than older subjects or those with significant psychopathology. A cumulative experience by this group of 250 patients has resulted in a response rate of $80 \% .{ }^{53}$

The mode of action of this treatment is unclear but it seems to be essential for the hypnosis to be 'gut directed'. A simple account of smooth muscle physiology is given before hypnosis is induced by an eye fixation and arm levitation technique followed by standard deepening procedures. Under hypnosis the patient is asked to place a hand on the abdomen, feel a sense of warmth and relate this to asserting control over gut function. When hypnosis is confined to emphasizing general relaxation without being directed towards control of intestinal function IBS symptoms do not improve. ${ }^{48}$ Although hypnotherapy is likely to produce psychological and/or placebo effects, improvement in IBS symptoms was not correlated with changes in anxiety or depression..$^{53}$ The same group has demonstrated that abnormal rectal sensitivity can be modified towards normal by hypnosis. ${ }^{54}$ This finding was not accompanied by any change in ability to withstand somatic pain.

\section{Group therapy}

Approximately 500 new patients present to gastroenterologists in the United Kingdom each week ${ }^{55}$ and up to $32 \%$ patients remain troubled by their symptoms in the long term, despite reassurance, antispasmodics and bulking agents. ${ }^{56}$ Alternative 
treatments such as hypnotherapy or psychotherapy if carried out on an individual basis are extremely time consuming. However, hypnotherapy in groups of up to 8 patients has been shown to be as effective as individual therapy ${ }^{57}$ and group stress management can also be helpful. ${ }^{58,59}$

\section{Food exclusion diets}

The role of food intolerance in the aetiology of IBS, and exclusion diets in its management remains controversial. An initial study showing two thirds of patients improved ${ }^{31}$ has received some recent corroboration $^{60}$ but placebo response may be an important factor influencing these results. Other studies have not confirmed the high response rate to this form of treatment and any improvement was confined to those with diarrhoea. ${ }^{61}$

\section{Conclusions}

The majority of patients will respond to a positive attitude of explanation, understanding and re-

\section{References}

1. Harvey, R.F., Salih, S.Y. \& Read, A.E. Organic and functional disorders in $\mathbf{2 0 0 0}$ gastroenterology outpatients. Lancet 1983 , i: $632-634$.

2. Thompson, W.G. The irritable bowel: one disease, or several, or none? In: Read, N.W. (ed.) Irritable Bowel Syndrome. Grune and Stratton, Philadelphia, 1985, 3-16.

3. Manning, A.P., Thompson, W.G., Heaton, K.W. \& Morris, A.F. Towards positive diagnosis of the irritable bowel syndrome. Br Med J 1978, 2: 653-654.

4. Whitehead, W.E., Crowell, M.D., Bosmajian, L. et al. Existence of irritable bowel syndrome supported by factor analysis of symptoms in two community samples. Gastroenterology 1990, 98: 336-340.

5. Thompson, W.G. \& Heaton, K.W. Functional bowel disorders in apparently healthy people. Gastroenterology 1980 , 79: $283-288$.

6. Drossman, D.A., Sandler, R.S., McKee, D.C. \& Lovitz, A.J. Bowel patterns among subjects not seeking health care. Use of a questionnaire to identify a population with bowel dysfunction. Gastroenterology 1982, 83: 529-534.

7. Langeluddecke, P.M. Psychological aspects of irritable bowel syndrome. Aust NZ J Psychiatry 1985, 19: 218-226.

8. Smith, R.C., Greenbaum, D.S., Vancouver, J.B. et al. Psychosocial factors are associated with health care seeking rather than diagnosis in irritable bowel syndrome. Gastroenterology 1990, 98: 293-301.

9. Welch, G.W., Hillman, L.C. \& Pomare, E.W. Psychoneurotic symptomatology in the irritable bowel syndrome: a study of reporters and non-reporters. Br Med J 1985, 281: 1382-1384.

10. Sandler, R.S., Drossman, D.A., Nathan, H.P. \& McKee, D.C. Symptom complaints and health care seeking behaviour in subjects with bowel dysfunction. Gastroenterology 1984, 87: $314-318$.

11. Drossman, D.A., McKee, D.C., Sandler, R.S. et al. Psychological factors in the irritable bowel syndrome. Gastroenterology 1988, 95: 701-708. assurance combined with appropriate use of dietary fibre, bulking agents and antispasmodics. A trial of a bile acid binding resin is worthwhile in patients with frank diarrhoea but psychotropic drugs should be reserved for those with evidence of an affective disorder. In the minority of patients who do not respond to these measures and continue with troublesome symptoms, the local availability of psychotherapy, stress management counselling or dietician back-up for exclusion diet supervision may determine which of these alternative treatments is pursued. If the excellent results of hypnotherapy are confirmed from other centres this may become the treatment of choice for refractory cases especially in a group setting. As knowledge of the enteric nervous system increases, pharmacological agents targeted to this area may eventually offer rational and effective therapy.

12. Whitehead, W.E., Bosmajian, L., Zonderman, A.B., Costa P.T. Jr \& Schuster, M.M. Symptoms of psychologic distresø associated with irritable bowel syndrome. Comparison community and medical clinic samples. Gastroenterolog 1988, 95: 709-714.

13. Heaton, K.W. IBS in the community. In: Towards Confident Management of the Irritable Bowel Syndrome. Symposium. Royal College of Physicians, London, 1990.

14. Ritche, J. Pain from distension of the pelvic colon by inflating a balloon in the irritable colon syndrome. Gut 1973, 14: 125-132.

15. Whitehead, W.E., Holtkotter, B., Enck, P. et al. Tolerance for rectosigmoid distension in irritable bowel syndrome. Gastroenterology 1990, 98, 1187-1192.

16. Cook, I.J., van Eeden, A. \& Collins, S.M. Patients with irritable bowel have greater pain tolerance than normal subjects. Gastroenterology 1987, 93: 727-733.

17. Lasser, R.B., Bond, J.H. \& Levitt, M.D. The role of intestinal gas in functional abdominal pain. $N$ Engl J Med 1975, 293. 524-526.

18. Prior, A., Maxton, D.B. \& Whorwell, P.J. Anorectal manometry in irritable bowel syndrome: differences between diarrhoea and constipation predominant subjects. Gut 1990 31: 458-462.

19. Ford, M.J. The irritable bowel syndrome. J Psychosom Res 1986, 30: 399-410.

20. Snape, W.J., Carlson, G.M. \& Cohen, S. Colonic myoelectric activity in the irritable bowel syndrome. Gastroenterology 1976, 70: 326-341.

21. Sarna, S., Latimer, P., Campbell, D. \& Waterfall, W. Effect of stress, meal and neostigmine on rectosigmoid electrical control activity (ECA) in normals and in irritable bowel syndrome patients. Dig Dis Sci 1982, 27: 582-591. 
22. Rogers, J., Henry, M.M. \& Misiewicz, J.J. Increased segmental activity and intraluminal pressures in the sigmoid colon of patients with the irritable colon syndrome. Gut 1989, 30: 634-641.

23. Thompson, G.D., Wingate, D.L., Archer, L., Benson, M.J., Green, W.J. \& Hardy, R.J. Normal patterns of human upper small bowel motor activity recorded by prolonged radiotelemetry. Gut 1980, 21: 500-506.

24. Kumar, D. \& Wingate, D.L. The irritable bowel syndrome: a paroxysmal motor disorder. Lancet 1985, ii: 973-977.

25. Kellow, J.E. \& Phillips, S.F. Altered small bowel motility in irritable bowel syndrome is correlated with symptoms. Gastroenterology 1987, 92: 1885-1893.

26. Mendeloff, A.I., Mark, M., Seigel, C.I. \& Lillienfield, A. Illness experience and life stresses in patients with irritable colon and ulcerative colitis. $N$ Engl J Med 1970, 282: 14-17.

27. Camilleri, M. \& Neri, M. Motility disorders and stress. Dig Dis Sci 1989, 34: 1777-1786.

28. Almy, T.P., Hinkle, L.E., Berle, B. \& Kern, F. Alterations in colonic function in man under stress. III. Experimental production of sigmoid colon spasm in patients with spastic constipation. Gastroenterology 1949, 12: 437-449.

29. Narducci, F., Snape, W.J., Battle, W.M., London, R.L. \& Cohen, $\mathrm{S}$. Increased colonic motility during exposure to a stressful situation. Dig Dis Sci 1985, 30: 40-44.

30. Merrick, M.V., Eastwood, M.A. \& Ford, M.J. Is bile acid malabsorption underdiagnosed? An evaluation of accuracy of diagnosis by measurement of the SeHCAT retention. $\mathrm{Br}$ Med J 1985, 290: 665-668.

31. Jones, V.A., McLaughlan, P., Shorthouse, M., Workman, E. \& Hunter, J.O. Food intolerance: a major factor in the pathogenesis of irritable bowel syndrome. Lancet 1982, ii: $1115-1117$.

32. Haderstorfer, B., Psychologin, D., Whitehead, W.E. \& Schuster, M.M. Intestinal gas production from bacterial fermentation of undigested carbohydrate in irritable colon syndrome. Am J Gastroenterol 1989, 84: 375-378.

33. Read, N.W. Irritable bowel syndrome (IBS) - definition and pathophysiology. Scand J Gastroenterol 1987, 130 (Suppl): 7-13.

34. Almy, T.P. \& Rothstein, R.I. Irritable bowel syndrome: classification and pathogenesis. Ann Rev Med 1987, 38: 257-265

35. Wingate, D. Motility disorders of the small intestine in functional intestinal disorders. Presse Med 1989, 18: 290-293.

36. Kellow, J.E. \& Langeluddecke, P.M. Advances in the understanding and management of the irritable bowel syndrome. Med J Aust 1989, 151: 94-99.

37. Manning, A.P., Heaton, K.W., Harvey, R.F. \& Uglow, P. Wheat fibre and irritable bowel syndrome. Lancet 1977, i: 417-418

38. Lucey, M.R., Clark, M.L., Lowndes, J. \& Dawson, A.M. Is bran efficacious in irritable bowel syndrome? A double blind placebo controlled crossover study. Gut 1987, 28: 221-225.

39. Prior, A. \& Whorwell, P.J. Double blind study of ispaghula in irritable bowel syndrome. Gut 1987, 28: 1510-1513.

40. Cann, P.A., Read, N.W. \& Holdsworth, C.D. What is the benefit of coarse wheat bran in patients with irritable bowel syndrome? Gut 1984, 24: 168-173.

41. Cook, I.J., Irvine, E.J., Campbell, D., Shannon, S., Reddy, S.N. \& Collins, S.M. Effect of dietary fiber on symptoms and rectosigmoid motility in patients with irritable bowel syndrome. A controlled crossover study. Gastroenterology 1990 , 98: $66-72$.
42. Klein, K.B. Controlled treatment trials in the irritable bowel syndrome: a critique. Gastroenterology 1988, 95: 232-241.

43. Centonze, V., Imbimbo, B.P., Campanozzi, F., Attolini, E., Daniotti, S. \& Albano, O. Oral cimetropium bromide, a new antimuscarinic drug for long term treatment of irritable bowel syndrome. Am J Gastroenterol 1988, 83: 1262-1266.

44. Mathias, J.R., Ferguson, K.L. \& Clench, M.H. Debilitating 'functional' bowel disease controlled by leuprolide acetate, gonadotrophin-releasing hormone (GnRH) analog. Dig Dis Sci 1989, 34: 761-766.

45. Creed, F. \& Guthrie, E. Psychological treatments of the irritable bowel syndrome: a review. Gut 1989, 30: 1601-1609.

46. Hislop, I.G. Effect of very brief psychotherapy on the irritable bowel syndrome. Med J Aust 1980, 2: 620-623.

47. Svedlund, J., Sjodin, I., Ottoson, J.O. \& Dotevall, G. Controlled study of psychotherapy in irritable bowel syndrome. Lancet 1983, ii: 589-592.

48. Whorwell, P.J., Prior, A. \& Faragher, E.B. Controlled trial of hypnotherapy in the treatment of severe refractory irritable bowel syndrome. Lancet 1984, ii: 1232-1234.

49. Guthrie, E. Psychotherapy with patients with the irritable bowel syndrome. In: Towards Confident Management of the Irritable Bowel Syndrome. Symposium. Royal College of Physicians, London, 1990

50. Bennet, P. \& Wilkinson, S. A comparison of psychological and medical treatment of the irritable bowel syndrome. $\mathrm{Br} J$ Clin Psychol 1985, 24: 215-216.

51. Blanchard, E.B., Schwarz, S.P., Neff, D.F. \& Gerardi, M.A. Prediction of outcome from the self regulatory treatment of irritable bowel syndrome. Behav Res Ther 1988, 26: 187-190.

52. Whorwell, P.J., Prior, A. \& Colgan, S.M. Hypnotherapy in severe irritable bowel syndrome: further experience. Gut 1987, 28: 423-425.

53. Whorwell, P.J. Use of hypnotherapy in gastrointestinal disease. Br J Hosp Med 1991, 45: 27-29.

54. Prior, A., Colgan, S.M. \& Whorwell, P.J. Changes in recta sensitivity following hypnotherapy for irritable bowel syndrome. Gut 1990, 31: 896-898.

55. Lennard-Jones, J.E. The need for an increased number of consultant physicians with specialist training in gastroenterology. Gut 1984, 25: 99-102.

56. Harvey, R.F., Mauad, E.C. \& Brown, A.M. Prognosis in the irritable bowel syndrome: a 5-year prospective study. Lancet 1987 , i: $963-965$.

57. Harvey, R.F., Hinton, R.A., Gunary, R.M. \& Barry, R.E. Individual and group hypnotherapy in treatment of refractory irritable bowel syndrome. Lancet 1989, i: 424-425.

58. Barreiro, M.A., Bank, L. \& Gitchell, L. Irritable bowel support group: a new form of management. J Clin Gastroenterol 1989, 11: 118-119.

59. Rumsey, N. Group stress management - a viable alternative to drugs? In: Towards Confident Management of the Irritable Bowel Syndrome. Symposium. Royal College of Physicians, London, 1990.

60. Nanda, R., James, R., Smith, H., Dudley, C.R. \& Jewell, D.P. Food intolerance and the irritable bowel syndrome. Gut 1989 , 30: 1099-1104.

61. McKee, A.M., Prior, A. \& Whorwell, P.J. Exclusion diets in irritable bowel syndrome: are they worthwhile? J Clin Gastroenterol 1987, 9: 526-528. 